\title{
The involvement of central attention in visual search is determined by task demands
}

\author{
Suk Won Han ${ }^{1}$
}

Published online: 23 January 2017

(C) The Psychonomic Society, Inc. 2017

\begin{abstract}
Attention, the mechanism by which a subset of sensory inputs is prioritized over others, operates at multiple processing stages. Specifically, attention enhances weak sensory signal at the perceptual stage, while it serves to select appropriate responses or consolidate sensory representations into short-term memory at the central stage. This study investigated the independence and interaction between perceptual and central attention. To do so, I used a dual-task paradigm, pairing a four-alternative choice task with a visual search task. The results showed that central attention for response selection was engaged in perceptual processing for visual search when the number of search items increased, thereby increasing the demand for serial allocation of focal attention. By contrast, central attention and perceptual attention remained independent as far as the demand for serial shifting of focal attention remained constant; decreasing stimulus contrast or increasing the set size of a parallel search did not evoke the involvement of central attention in visual search. These results suggest that the nature of concurrent visual search process plays a crucial role in the functional interaction between two different types of attention.
\end{abstract}

Keywords Central attention · Perceptual attention · Visual search

The human brain is constantly bombarded with massive amounts of information. However, despite the impressive capability and complexity of the brain, the capacity of human

Suk Won Han

suk.w.han@gmail.com

1 Department of Psychology, Chungnam National University, Daejeon, Republic of Korea information processing is severely limited, such that only a subset of sensory inputs can be consciously perceived, acted upon, or stored in memory (Cohen, Cavanagh, Chun, \& Nakayama, 2012; Marois \& Ivanoff, 2005). Under these capacity limits, selective attention, by which a subset of information is prioritized or processed in finer detail at the expense of other information, plays a crucial role to protect the system from information overload and optimally allocates limited processing resources (Desimone \& Duncan, 1995; Prinzmetal, McCool, \& Park, 2005).

A predominant theory of capacity limitations, the central bottleneck model, has proposed that there are distinct classes of attention, each of which operates at different processing stages (Pashler, 1984, 1991). Specifically, the performance of any sensorimotor task involves distinct stages of processing, namely, perceptual, central, and motor stages (see Fig. 1). At the perceptual stage, where the extraction of sensory data and identification of the stimuli occurs, attention operates to enhance sensory signals and distinguish them from noise (Carrasco, Penpeci-Talgar, \& Eckstein, 2000; Dosher \& Lu, 2000; Han \& Marois, 2014; Luck \& Ford, 1998). At the central stage, attention plays a role in the process of response selection or short-term memory consolidation followed by the execution of selected response at the motor stage (Jolicoeur \& Dell‘Acqua, 1998; Pashler, 1984, 1994a; Tombu \& Jolicoeur, 2003, 2005).

The central bottleneck model posits that there is a bottleneck at the central stage, allowing only a single central operation to proceed at a time. This account provides a simple explanation for an illustrative example of capacity limitations, the psychological refractory period phenomenon (PRP); when people try to perform two sensorimotor tasks in rapid succession, the response to the second task is almost invariably delayed (Welford, 1952). According to the model, the PRP originates because the response selection of the second-presented 
a

long SOA
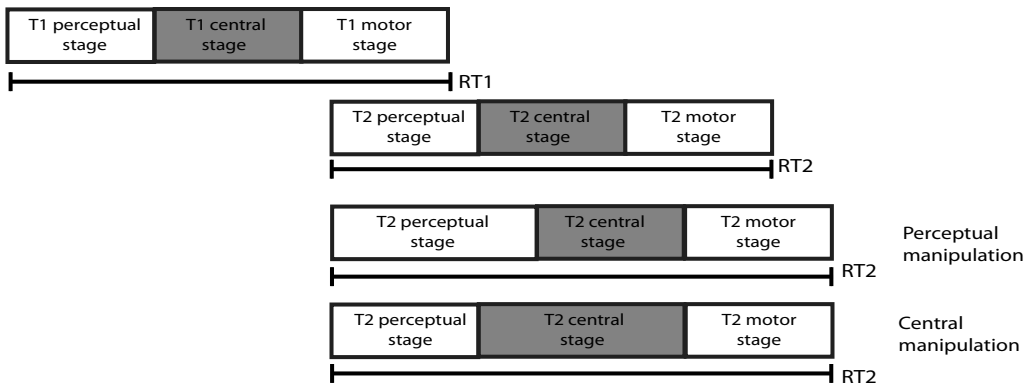

b

short SOA

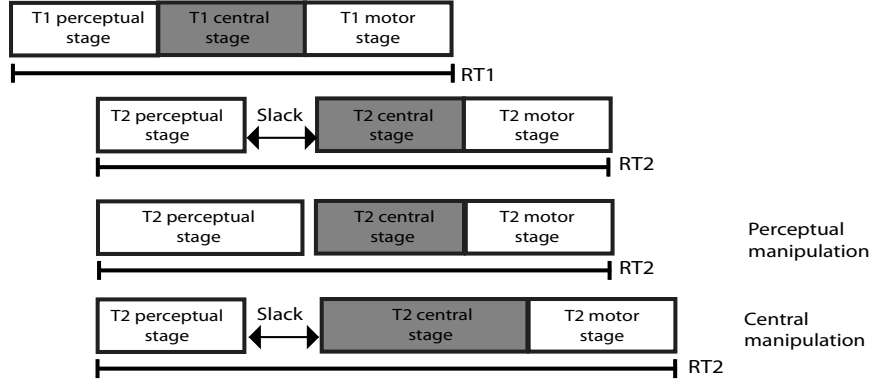

C

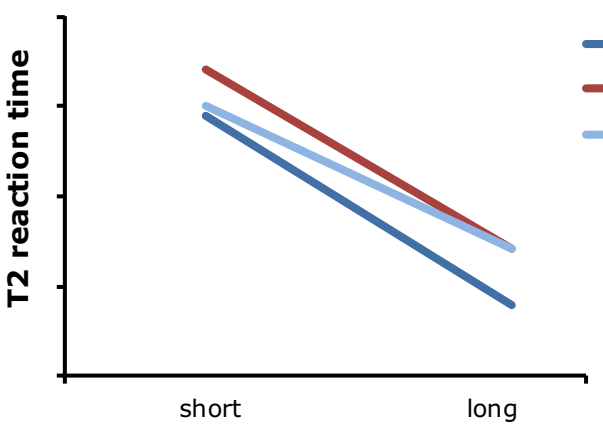

Fig. 1 Processing stages and differential effects of lowering stimulus contrast and increasing the number of stimuli. a At the long SOA, both the central and perceptual manipulations slows Task 2 RT. b At the short $\mathrm{SOA}$, the increased duration of the perceptual process is absorbed into the cognitive slack period produced by the overlap between the two central

task should be postponed until the first response selection is completed (Pashler, 1984, 1994a; but see also Tombu \& Jolicoeur, 2003, 2005).

Another important proposition of the central bottleneck model is that even though two separate response selections cannot proceed simultaneously, the perceptual process of a stimulus can take place simultaneously with the central process of another stimulus without interference. This is because the attentional mechanisms involved in each stage of processing have separate, independent capacities. While the strict bottleneck account has been challenged by other models for the PRP, such as the central capacity sharing model (Tombu \& Jolicoeur, 2003, 2005), it is commonly agreed that the central processes, whereas the increased central processing duration is not. c Predicted results for Task 2 RT. Perceptual and central manipulations should have an underadditive and an additive effect on Task 2 RT with SOA, respectively. $\mathrm{T} 1=$ Task $1 ; \mathrm{T} 2=$ Task 2

stage is the locus of the PRP and has separate capacity from the perceptual stage.

The claim that central and perceptual attention are independent has been empirically supported by a number of studies showing that perceptual processing is not subject to the central capacity limitation of response selection (Johnston, McCann, \& Remington, 1995; Pashler \& Johnston, 1989). In those studies, applying the locus of slack method (Schweickert, 1980), participants were required to make immediate and speeded responses to two stimuli, which were separated by a short or long stimulus onset asynchrony (SOA). An important experimental factor was perceptual difficulty of the second task; while the demand of the first task (Task 1, T1) remained 
the same, the second task (Task 2, T2) stimuli were distorted or their contrast was lowered. Obviously, this perceptual manipulation of T2 stimuli lengthened T2 RT when the SOA between $\mathrm{T} 1$ and $\mathrm{T} 2$ was long enough that each task could be performed in isolation. By contrast, with the short SOA, such T2 manipulation affected T2 RT little, yielding an underadditive interaction of SOA and T2 difficulty on $\mathrm{T} 2$ RT. This was because the increased processing duration of $\mathrm{T} 2$ by distorting or contrast-lowering of the stimuli was absorbed into a cognitive slack period. Specifically, as shown in Fig. 1b, with the short SOA, the central processes of T1 and T2 overlap. This overlap postpones the central process of T2 until the $\mathrm{T} 1$ central process finishes, which produces a slack period. However, the perceptual process of $\mathrm{T} 2$ can be done in parallel with the $\mathrm{T} 1$ central process. Thus, when perceptual processing of $\mathrm{T} 2$ was lengthened, the increased processing duration could be absorbed into the slack period.

Notably, not all kinds of $\mathrm{T} 2$ difficulty manipulations have underadditive effects on the perceptual task RT with SOA. The processes of mental rotation, spatial position judgement, size scaling, and other high-level classifications (e.g., lexical decision) were found to require some processing at the central stage (Johnston \& McCann, 2006; McCann, Remington, \& Van Selst, 2000; Ruthruff, Miller, \& Lachmann, 1995). While these results suggest that besides response selection there are certain classes of processes taking place at the central stage, the process of identifying prespecified target stimuli seems to take place primarily at the perceptual, precentral stage.

Expanding these previous studies, a recent study systematically varied the demand for perceptual attention needed for a visual task and tested the possibility of interaction between perceptual and central attention (Reimer, Strobach, Frensch, \& Schubert, 2015). In this study, participants performed an auditory discrimination task and a visual attention task, which were separated by variable SOAs. The visual task consisted of searching for a target stimulus defined by the combination of a specific color and an orientation (red vertical bar) among a variable number of distractors (red horizontal and green vertical bars). What is novel in this study was that the demand for perceptual attention was increased by increasing the number of search items rather than degrading the stimulus quality or increasing the complexity of the target process.

The results showed that increasing the search set size had an underadditive effect on search RT with the SOA between the two tasks; search RT increased as a function of search set size only with the long SOA. The same pattern of results was found when the visual search task was preceded by another visual task (color discrimination task). Based upon these, they concluded that visual attention deployed for conjunction search was independent from central attention needed for response selection.

However, contrary to that study, other researchers reported findings that the deployment of visuospatial attention for visual search was impaired by concurrent central processing (Brisson \& Jolicoeur, 2007a, b; Robitaille, Jolicoeur, Dell'Acqua, \& Sessa, 2007). In such a study (Brisson \& Jolicoeur, 2007a), participants performed a tone discrimination task and a visual search task, which were separated by a short or a long SOA. While participants were doing the tasks, their electrophysiological responses were recorded. As a result, at the short SOA, the N2pc component of event-related potential (ERP), a negative deflection of the ERP at posterior electrodes contralateral to the position where focal attention is allocated, was attenuated. Given that the N2pc is a neural marker of the deployment of visuospatial attention toward a specific location (Woodman \& Luck, 1999), this result suggests that response selection load taxing central attention impaired the deployment of focal attention necessary for perceptual processing. In line with this, a study by Lien and colleagues also demonstrated the attenuation of the N2pc component by concurrent response selection load (Lien, Croswaite, \& Ruthruff, 2011).

How can these contradicting results be reconciled? Based on the studies using the cognitive slack paradigm, it is quite clear that the perceptual identification process of a stimulus can proceed simultaneously with the central process of another stimulus. Thus, perceptual and central attention seem to be independent. However, there also seems to be cases when central attention is involved in perceptual processing. First, given the findings by Brisson and Jolicoeur and those by Lien and colleagues, the demand for response selection should be substantial; these studies showed that the performance of fourchoice tasks interfered with the immediate deployment of spatial attention (Brisson \& Jolicoeur, 2007a), while the twochoice task did not (Lien et al., 2011). Second, the performance of the search task immediately following the response selection task should require the allocation of focal attention to a specific stimulus/location. That is, central attention seems to play a role in the deployment of focal attention when it is needed for perceptual processing (Brisson \& Jolicoeur, 2007a). One can thus predict that when the performance of a given search task requires the serial allocation of focal attention, concurrent response selection load should interfere with the search process. By contrast, when multiple search stimuli are processed in parallel without multiple deployments of focal attention, the search should be able to proceed simultaneously with the response selection process without impairment.

Reimer and colleagues assumed that conjunction search is performed by serially shifting focal attention among the search items (Reimer et al., 2015). This assumption is grounded on a seminal study by Treisman and Gelade (1980). This study showed that when participants searched for a singleton target, search performance was invariant, regardless of how many search items were presented. By contrast, when participants searched for a conjunction target, formed by combining two different features, search performance suffered as the number of items increased. These findings were explained 
by positing that the conjunction search requires capacitylimited attentional resource. Specifically, to detect a combination of multiple features among heterogeneous distractors, focal attention should be serially allocated to each search item. This serial search is inevitable because the amount of attentional resource deployed for visual search is finite.

However, the strict distinction between serial and parallel searches has long been challenged. According to the guided search model proposed by Wolfe and colleagues (Wolfe, Cave, \& Franzel, 1989), serial search is needed to ultimately locate the target. However, prior to serial shifting of attention, parallel processing of multiple items takes place to guide focal attention. Furthermore, some researchers even demonstrated that a type of conjunction search (size-orientation conjunction) proceeded in an automatic, capacity-unlimited manner, obviating the need for serial shifting of attention (Huang \& Pashler, 2005).

While another study showed that the process of binding two simple features might require capacity-limited attentional resources (Bouvier \& Treisman, 2010), unequivocal evidence showing that conjunction search is performed in a serial manner is lacking. Contrary to the claims of Treisman and Gelade (1980) and Wolfe and colleagues (1989) that serial attention is need for detecting a combination of features, several groups of researchers have found evidence that parallel processing of multiple items is sufficient for detecting a conjunction target (Bichot, Rossi, \& Desimone, 2005; Sung, 2008). For example, in the process of searching for a red vertical bar among green vertical and red horizontal bars, any green item should be filtered out, and only the red items should be candidates for further processing. In line with this, an anonymous reviewer also pointed out that the performance of conjunction search should include such a process of tagging the locations containing the target color items. Then, this parallel activation of the target color locations should guide focal attention toward the target location.

It is important to note that even though multiple items can be processed in parallel, it is possible that the search process is still capacity limited. If this is so, as mentioned above, limited amount of attentional resource should be shared by multiple items (Sung, 2008; Townsend, 1971, 1972). Hence, as the number of search items increases, processing rate of each item should decrease, yielding a steep search slope. Specifically, I suggest that the feature-based, location-tagging process takes place in parallel, but consumes the limited perceptual capacity. In this framework, increasing the set size of a conjunction search task, even though that manipulation increases the demand for attentional resources distributed among the search items, does not increase the demand for serially shifting the focal attention. This might be why the set size manipulation of conjunction search had an underadditive effect on search RT with SOA.

I do not argue that conjunction search does not depend on focal attention. In the process of reporting the presence/ absence or identifying a conjunction target stimulus, focal attention has to be allocated to the target location (Bichot et al., 2005). What matters here is whether the number of deployment of focal attention would increase as set size increases. For conjunction search, this would not be the case; multiple items are analyzed in parallel in the way elaborated above, followed by the deployment of focal attention toward the located target stimulus.

To be clear, even though I suggest an alternative explanation to the conventional serial search account about how conjunction search is performed, I do not make any strong claim regarding this issue. What seems to be clear is that the performance of conjunction search depends on set size. However, there are at least two possible causes for the set size effect; it might be either due to serial search or due to limited parallel search. It is also noteworthy that a study showed that a type of conjunction search (size-orientation conjunction), yielding significant search slope, did not depend on capacity-limited attentional resources (Huang \& Pashler, 2005).

Contrary to the case of conjunction search, if a search task that is unequivocally known to be performed in a serial manner (e.g., an orientation target search task; see Buschman \& Miller, 2009; Woodman \& Luck, 1999) is paired with a response selection task, a different result should emerge. In this case, increasing search set size would also increase the demand for serially allocating focal attention. Given the evidence that the allocation of focal attention toward a specific location/stimulus is mediated by central attention (Brisson \& Jolicoeur, 2007a), set size and SOA should yield an additive effect on search RT when the search proceeds serially.

To test this prediction, I designed an experiment employing the cognitive slack method. In the experiment, participants performed a dual task, consisting of a letter identification task (Task 1, T1) and a visual search task (Task 2, T2). It is important to note that given the purpose of this study, I intended to choose visual tasks for both $\mathrm{T} 1$ and $\mathrm{T} 2$ to maximize the interference between the tasks (Reimer et al., 2015). Another important point is that for T2, I chose a task of searching for a specific orientation target among heterogeneous distractors because this kind of search is known to require the deployment of focal attention (Buschman \& Miller, 2009; Wolfe, FriedmanHill, Stewart, \& O'Connell, 1992; Woodman \& Luck, 1999).

In each trial of this experiment, $\mathrm{T} 1$ was followed by $\mathrm{T} 2$ with a short or long SOA. T2 difficulty was manipulated in two different ways. In one type of trials, the number of T2 stimuli increased. With this manipulation, behavioral performance suffers because processing load increased, straining the perceptual capacity. In the face of this capacity limitation, focal attention would have to be serially allocated to each item. In the other type of trials, the contrast of T2 stimuli was lowered. This manipulation has been known to affect the visual task performance at the perceptual, but not at the central stage (Pashler \& Johnston, 1989). I included this contrast-lowering manipulation as a way of confirming that the current experimental paradigm can replicate a well-established pattern of result. 
To predict results, when the contrast of stimuli is lowered, T2 RT at the long SOA should be lengthened, but T2 RT at the short SOA should not be affected. This is because the increased processing duration at the perceptual stage can be absorbed into a slack period evoked by overlap between the two tasks (see Fig. 1b). Hence, when the contrast of T2 stimuli is lowered, an underadditive interaction of the SOA and perceptual difficulty on T2 RT should be found (Johnston et al., 1995; Pashler \& Johnston, 1989). By contrast, when the set size of the orientation search task increases, the demand for focal attention should increase, evoking the involvement of central attention. In this case, the duration of central processing should be lengthened, yielding an additive effect of SOA and perceptual difficulty on T2 RT. That is, T2 RT should be lengthened both at the short and at the long SOAs (see Fig. 1c).

\section{Experiment 1}

\section{Method}

\section{Participants}

Eighteen adults (eight males, 18-25 years) with normal or corrected-to-normal vision participated for course credit. Prior to running the main experiment, I performed a pilot test to determine the appropriate sample size. The power analysis based upon the pilot testing revealed that $N$ of 14 should be enough to detect a significant effect at the level of .80 . Informed consent was obtained from each participant.

\section{Stimuli and apparatus}

The experiment was programmed and run using PsychoPy (Peirce, 2007). The stimuli were presented on a 21-in. LCD monitor with a gray background. The first task (T1) was a letter identification task. Participants responded to four different Korean letters, 가, 나, 다, or 라 by pressing z, x, c, or v buttons on a keyboard with their left hand. The letters were written in black and presented at the center of the screen. The height of letters was 1 degree of visual angle.

The second task (T2) was a visual search task. The search stimuli were Gabor gratings $\left(1.5^{\circ} \times 1.5^{\circ}\right)$, presented on an imaginary circle around the center of the screen, whose radius was 3 degrees. Participants were required to search for a 45 degree-tilted grating among vertical or horizontal gratings and indicate whether the target was tilted to the left or right by pressing "." or " "” buttons with their right hand. The contrast of the gratings was $15 \%$ or $100 \%$ (see below).

\section{Design and procedure}

As shown in Fig. 2, T1 stimulus was presented for $20 \mathrm{~ms}$, followed by the $230-\mathrm{ms}$ T2 stimuli presentation. The SOA

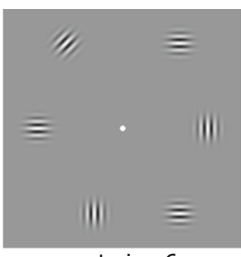

set size 6
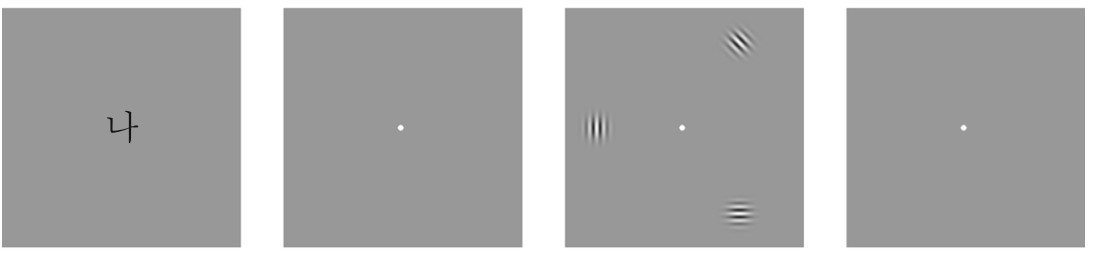

set size 3

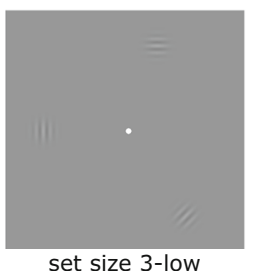

set size 3-low

$\begin{array}{rlrl}20 \mathrm{~ms} & 100 \text { or } 1800 \mathrm{~ms} & 230 \mathrm{~ms} & 500 \mathrm{~ms}\end{array}$

Fig. 2 Trial design of Experiment 1 
between the two tasks was short $(120 \mathrm{~ms})$ or long $(1,820 \mathrm{~ms})$. Participants were instructed to perform the two tasks as fast and accurately as possible. There were four levels of T2 task difficulty: Set Size 3, Set Size 3-low, Set Size 6, and Set Size 6-low. In the baseline trials (Set Size 3), three high-contrast $(100 \%)$ Gabor gratings were presented. One of them was a $45-$ degree-tilted target grating and the others were a vertical grating and a horizontal distractor grating. In the Set Size 3-low trials, three Gabor gratings were presented as in the Set Size 3 trials, but their contrast was low $(15 \%)$. In these trials, the quality of task stimuli was impoverished, but processing load should remain the same (Garner, 1970; Lavie \& de Fockert, 2003). Contrary to the Set Size 3-low trials, there were trials, in which task difficulty was increased by increasing the number of search items. In these Set Size 6 trials, six search items were presented, straining more processing capacity than in the Set Size 3 and Set Size 3-low trials. Finally, there were Set Size 6-low trials, in which six, low-contrast search stimuli were presented. All these different types of trials were randomly intermixed within an experimental block.

To summarize, the experiment consisted of a $2 \times 4$ factorial design with SOA (short and long) and T2 difficulty (Set Size 3, Set Size 3-low, Set Size 6, and Set Size 6-low) as withinsubject factors. A total of 576 trials were divided into six experimental blocks. There were 48 trials for each SOA ad $\mathrm{T} 2$ difficulty level. Because the main comparisons of interest were comparisons between the Set Size 3 and Set Size 3-low trials and between the baseline and Set Size 6 trials, an initial analysis did not include the data for Set Size 6-low trials were not analyzed. These trials were included in the experiment to balance the numbers of high- and low-contrast and Set Size 3and Set Size 6-trials. Hence, to analyze data, I applied a $2 \times 3$ repeated-measures ANOVA to reaction time (RT) and response accuracy data with SOA (short and long) and T2 difficulty (Set Size 3, Set Size 3-low, Set Size 6) as factors. Then, a subsequent analysis was based upon all the data, including the Set Size 6-low data. Significant main effects and interactions were further tested by $t$ tests. Statistical thresholds of these were corrected for multiple comparisons using False Discovery Rate procedure.

\section{Results and discussion}

Mean accuracies of the letter (T1) and search (T2) tasks were $98 \%$ and $95.5 \%$, respectively (see Table 1 ). A $2 \times 3$ repeatedmeasures ANOVA with SOA (short and long) and T2 difficulty (Set Size 3, Set Size 3-low, Set Size 6) as factors was applied to T1 and T2 accuracy data. These analyses revealed that there was a significant main effect of $\mathrm{T} 2$ difficulty on $\mathrm{T} 2$ accuracy, $F(2,34)=4.26, p<.05, \eta_{\mathrm{g}}^{2}=.05$. This was because search accuracy at the Set Size 6 trials $(94.2 \%)$ was significantly lower than at the Set Size 3 (96.5\%) and Set Size 3-low
Table 1 RT (ms) and accuracy (\%, in parentheses) data for Experiment 1

Task 2 (visual search task)

Task 2 difficulty

SOA Set Size $3 \quad$ Set Size $6 \quad$ Set Size 3-low Set Size 6-low

Short $\quad 1,008(95.8) \quad 1,061(94.3) \quad 1,002(95.0) \quad 1,045(92.6)$

Long $799(97.2) \quad 861(94.2) \quad 849(96.9) \quad 904(94.1)$

Task 1 (four-choice letter task)

Task 2 difficulty

SOA Set Size 3 Set Size 6 Set Size 3-low Set Size 6-low

Short $\quad 854(97.3) \quad 892(97.5) \quad 821(98.0) \quad 863(98.6)$

Long $\quad 542(98.1) \quad 544(99.0) \quad 544(98.6) \quad 547(98.7)$

trials $(96 \%), p \mathrm{~s}<.05$. No other main effect or interaction was found from the accuracy data. This high level of search accuracy and the absence of interaction between factors warrants that subsequent RT data analyses would not be confounded by speed-accuracy trade-offs.

The same analysis was applied to RT data (see Fig. 3). For T2 RT, there was a significant main effect of T2 perceptual difficulty, $F(2,34)=12.45, p<.01, \eta_{\mathrm{g}}^{2}=.038$. The main effect of SOA was also significant, $F(1,17)=90.78, p<$ $.01, \eta_{\mathrm{g}}^{2}=.28$. Most important, the interaction between SOA and T2 perceptual difficulty was significant, $F(2,34)=6.19, p$ $<.01, \eta_{\mathrm{g}}^{2}=.01$. To further investigate this interaction, I ran pairwise interaction analyses. When the data for Set Size 3 and Set Size 3-low trials were analyzed, there was a significant underadditive interaction between SOA and perceptual difficulty, $F(1,17)=11.68, p<.01, \eta_{\mathrm{g}}^{2}=.01$. Specifically, there was a significant RT increase at the long SOA $(49 \mathrm{~ms}, \mathrm{p}<.01)$, whereas no significant increase $(6 \mathrm{~ms})$ was found at the short SOA. This is a replication of well-established findings from studies using similar paradigms (Johnston et al., 1995; Pashler

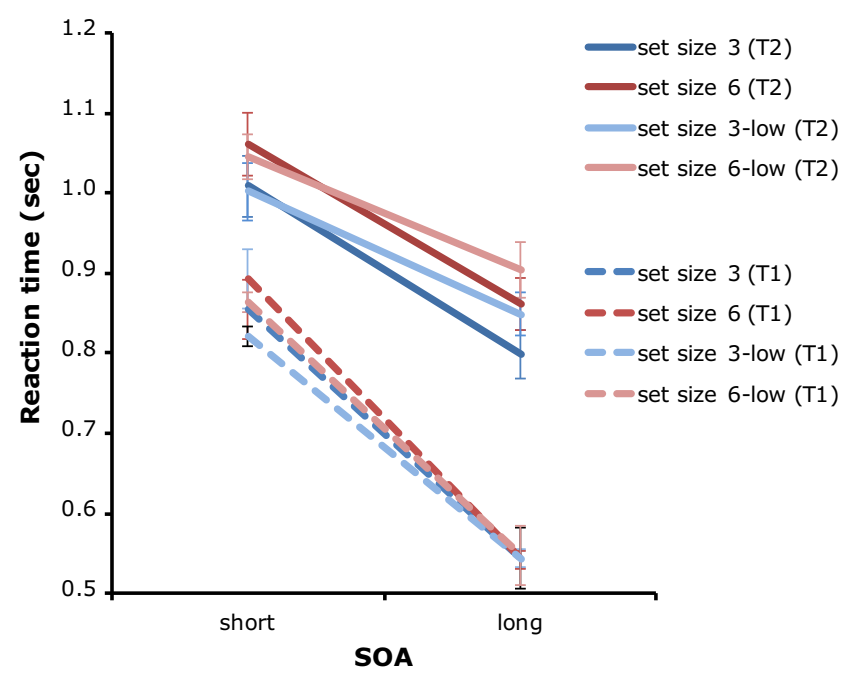

Fig. 3 Results of Experiment 1. Error bars represent standard errors of the mean. T1 $=$ Task $1 ; \mathrm{T} 2=$ Task 2 (Color figure online) 
\& Johnston, 1989); even though the current experimental settings were not conventional, regarding the adopted visual stimuli (Gabor gratings) and timing parameters (excessively different SOAs; but see Brisson \& Jolicoeur, 2007a), this finding provides an important validation of the present experiment and set up a stage for further analyses. By contrast, the analysis using the data for Set Size 3 and Set Size 6 trials revealed no such interaction, $p>.51$; similar RT increases were found at both SOAs as the search set size increases $(52 \mathrm{~ms}$ and $62 \mathrm{~ms}$ for the short and long SOAs, respectively).

Following the above analyses, similar analyses were run, using all the dataset, including the Set Size 6-low trial data. The results remained the same; the main effects of SOA, perceptual difficulty, and interaction were significant, $p \mathrm{~s}<.01$. Most important, the mean RT for the Set Size 6-low trial was significantly longer than that for Set Size 6 trial only at the long SOA, $t(17)=3.47, p<.01$, but not at the short SOA, $p$ $>$.0.27. No such interaction was found when the Set Size 3low and Set Size 6-low data were analyzed; the RT differences were significant both at the short and long SOAs, $p \mathrm{~s}<.01$, and these did not differ from each other (43 ms vs. $55 \mathrm{~ms}$ ), $p>.51$.

To summarize, the set size and contrast manipulations differentially affected the visual search RT. Increasing set size and lowering stimulus contrast similarly lengthened T2 RT at the long SOA, whereas at the short SOA, T2 RT was lengthened only when set size was increased. These results suggest that while contrast-lowering lengthens the processing duration at the perceptual stage, set size manipulation primarily affects central processing of the search.

While this study provides evidence that response selection load differentially interacts with the set size and contrast manipulations, an analysis of T1 RT revealed that there could be an alternative explanation for the current results. The ANOVA applied to the T1 RT data revealed significant main effects SOA, $F(1,17)=109.9, p<.01, \eta_{\mathrm{g}}^{2}=.66$, and T2 perceptual difficulty, $F(2,34)=20.56, p<.01, \eta_{\mathrm{g}}^{2}=.02$. More important, the interaction between SOA and perceptual difficulty was also significant, $F(2,34)=11.55, p<.01, \eta_{\mathrm{g}}^{2}=.02$.

The significant slowing of $\mathrm{T} 1$ response at the short SOA and the effect of $\mathrm{T} 2$ difficulty on $\mathrm{T} 1$ performance were also reported in many other studies (Brisson \& Jolicoeur, 2007a; Miller, Ulrich, \& Rolke, 2009; Pashler, 1991; Tombu \& Jolicoeur, 2003, 2005). Indeed, in a previous study, in which T1 was an auditory four-alternative choice task, a similar magnitude of T1 slowing with the current experiment was found (Brisson \& Jolicoeur, 2007a). While there is a debate regarding the causes of this T1 slowing (Han \& Marois, 2013b; Pashler, 1994b; Tombu \& Jolicoeur, 2003), it does not affect the interpretation of the observed pattern of T2 RT data. Specifically, the observed T1 slowing might be either because $\mathrm{T} 1$ and $\mathrm{T} 2$ share the central capacity or because participants grouped their responses. According to the central capacity sharing model, regardless of how much capacity is shared between $\mathrm{T} 1$ and $\mathrm{T} 2$, precentral manipulations of $\mathrm{T} 2$ has underadditive effects on T2 RT with SOA (Tombu \& Jolicoeur, 2003). Likewise, the presence/absence of response grouping was found to only affect T1 RT data, but had no effect on T2 RT data and their interpretation (Ulrich \& Miller, 2008); even when participants grouped their responses, precentral manipulation of $\mathrm{T} 2$ has an underadditive effect on T2 RT with SOA.

However, the significant interaction between SOA and perceptual difficulty on $\mathrm{T} 1$ should be seriously considered. Specifically, the interaction was because T1 RT was significantly longer for the Set Size 6 trials than for the Set Size 3 trials at the short SOA, $p<.01$, while there was no difference at the long SOA. This T1 RT increase at the short SOA might have been carried over to T2 RT. Specifically, it is possible that the set size manipulation had an underadditive effect with SOA, lengthening T2 RT only at the long SOA, but not at the short SOA. However, when T1 response at the short SOA was delayed, this would also delay $\mathrm{T} 2$ response, mimicking that set size and SOA had an additive effect, lengthening T2 RT at both SOAs.

To test this possibility, I ran a control analysis, in which a repeated measure 2 (short and long SOAs) $\times 2$ (Set Size 3 and Set Size 6) ANOVA was run, excluding data for participants showing excessively large T1 slowing at the Set Size 6 trials (see Fig. 4a). Given that a power analysis revealed that $N$ of 14 was sufficient to detect significant effect at the level of .80 , four participants' data were excluded. Now, as shown in Fig. 4a, there was no interaction between SOA and T2 perceptual difficulty on T1 RT, $p>.37$. The set size manipulation still had similar effects on T2 RT at both SOAs, $p>.51$. Other aspects of the result also remained the same; there were significant main effects of SOA, $F(1,13)=52.6, p<.01$, and perceptual difficulty, $F(1,13)=12.2, p<.01$. A similar analysis was also run to control for the effect of T2 contrast lowering on T1 RT (see Fig. 4b). The repeated measure 2 (short and long SOAs) $\times 2$ (Set Size 3 and Set Size 3-low) ANOVA revealed that contrast lowering had an underadditive effect on T2 RT with SOA, $F(1,13)=5.03, p<.05$. These results confirm that the observed pattern of results was not because T1 RT increase was carried over to T2 RT, but because response selection load differentially interacted with the set size and contrast manipulations.

Taken together, consistent with my prediction, as the number of items in the orientation search display increased, search RT was lengthened both at the short and long SOAs. This is because the performance of the search task requires the serial allocation of focal attention to the search stimuli. As the search set size increased, the demand for focal attention also increased. In this case, central attention was involved to efficiently shifting focal attention. By contrast, when multiple items could be processed in parallel during the search, increasing the search set size should not increase the demand for focal 


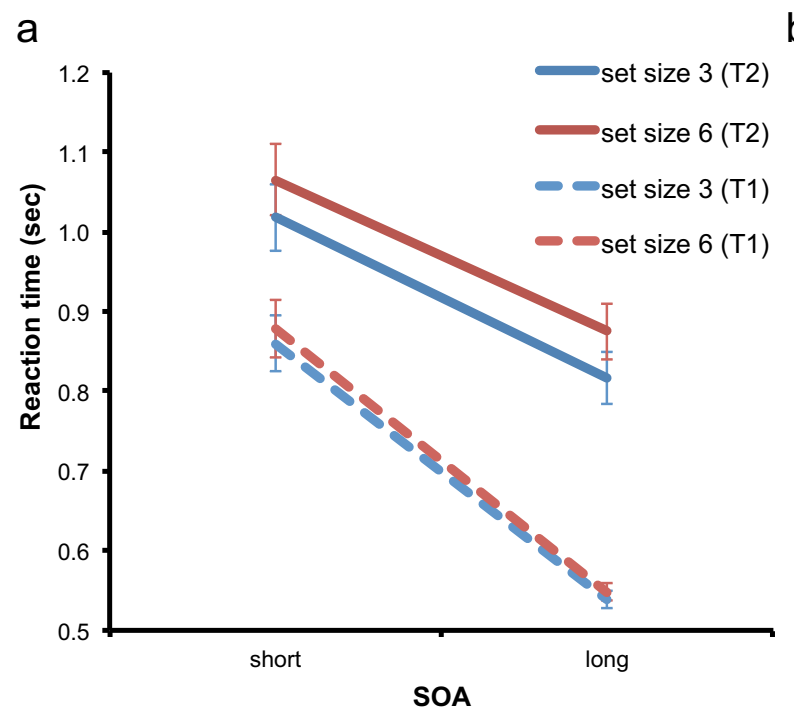

b

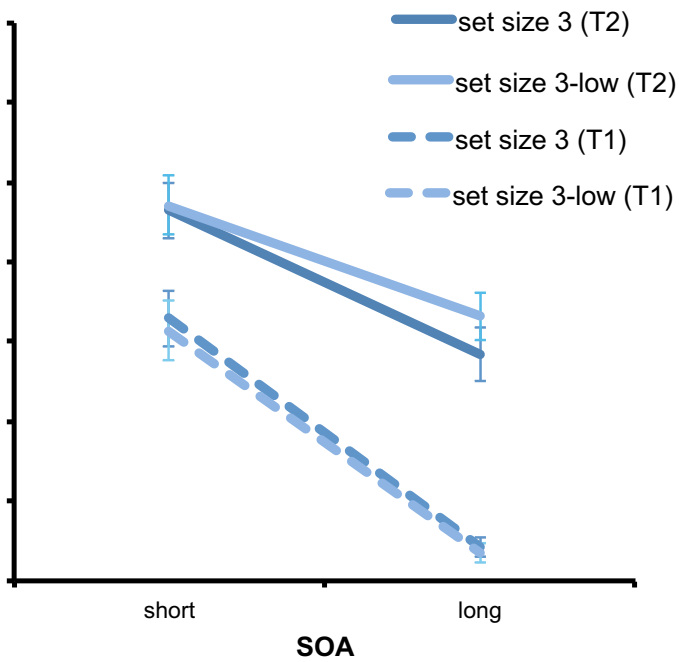

Fig. 4 Results of Experiment 1 data analyses controlling for the interaction of T2 difficulty and SOA on T1 RT under set size (a) and contrast manipulations (b). Error bars represent standard errors of the mean. T1 $=$ Task 1; T2 $=$ Task 2 (Color figure online)

attention. In this case, as Reimer and colleagues found, the set size manipulation should have no effect on search RT at the short SOA, but lengthen search RT at the long SOA.

\section{Experiment 2}

Experiment 1 showed that while perceptual and central attention were independent, central attention could be involved in perceptual processing required to perform visual search when the demand for focal attention increased. This is a contrasting result with the findings by Reimer and colleagues (Reimer et al., 2015). I argue that this is because the natures of employed search tasks were different; the orientation search in my Experiment 1 was performed by serially allocating focal attention to search items (Buschman \& Miller, 2009; Wolfe et al., 1992; Woodman \& Luck, 1999), whereas conjunction search in the study by Reimer and colleagues proceeded in parallel (Bichot et al., 2005; Sung, 2008). To further support this claim, in Experiment 2, I aimed at replicating the findings by Reimer and colleagues, using the similar experimental procedure and stimuli with those of Experiment 1. Most of the details of the procedure and stimuli were identical to those of Experiment 1, except that now participants performed a conjunction search task; the target was defined as a conjunction of a specific color and an orientation.

\section{Method}

\section{Participants}

Fourteen adults (nine males, 18-25 years) with normal or corrected-to-normal vision participated for course credit. Informed consent was obtained from each participant.

\section{Stimuli and apparatus}

The same stimuli and apparatus as Experiment 1 were used, except for the following. T1 letters were written in white against a black background. T2 was a conjunction search task. The target was a red, 45-degree-tilted grating, which was tilted to the left or right. The distractors comprised red vertical gratings and green 45 degree-tilted gratings. There were Set Size 3 and Set Size 6 trials.

\section{Design and procedure}

The details were identical to those of Experiment 1, except for the following. As shown in Fig. 5, while T1 remained the same, for T2, participants searched for a red 45-degree-tilted gratings among red vertical gratings and green 45-degree tilted gratings and indicated whether the target was tilted to the left or right via button presses. The design consisted of a 2 $\times 2$ factorial design with SOA (short and long) and set size (Set Size 3 and Set Size 6) of T2 as within-subject factors.

\section{Results and discussion}

Mean accuracies for T1 and T2 were $96.8 \%$ and $92.5 \%$, respectively (see Table 2). A repeated-measures two-way ANOVA applied to T1 and T2 accuracy data revealed that there were main effects of set size, $F(1,13)=40.2, p<.01$, $\eta_{\mathrm{g}}^{2}=.21$, and SOA, $F(1,13)=20.46, p<.01, \eta_{\mathrm{g}}^{2}=.17$, on T2 accuracy. No other main effect or interaction was significant.

The ANOVA applied to T2 RT data showed that the main effect of set size was significant, $F(1,13)=30.94, p<.01, \eta^{2}$ $=.02$, and so was the main effect of SOA, $F(1,13)=104.5, p$ $<.01, \eta_{\mathrm{g}}^{2}=.44$. Importantly, the interaction between SOA and 

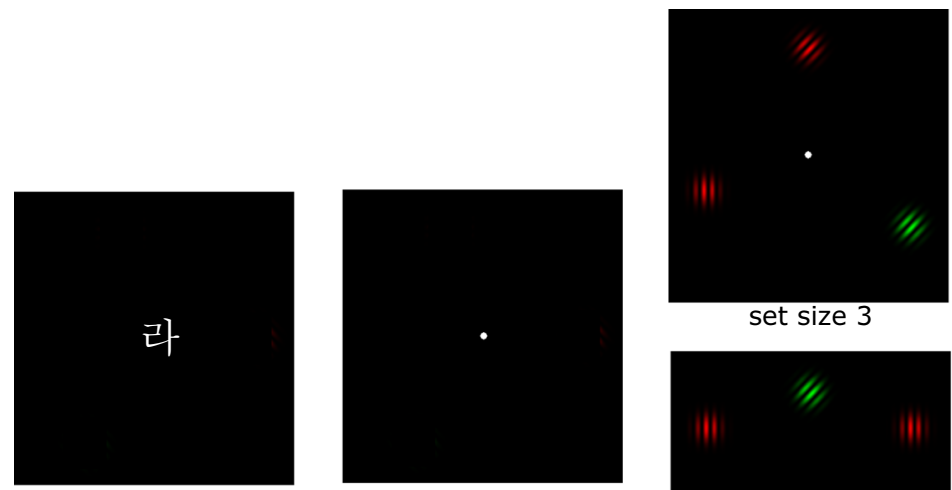

set size 3

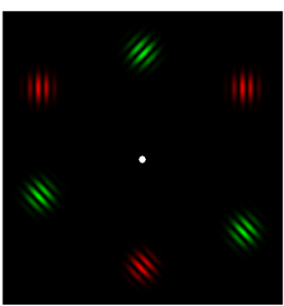

set size 6

$230 \mathrm{~ms}$

Time

Fig. 5 Trial design of Experiment 2

set size was also significant, $F(1,13)=24.57, p<.01, \eta_{\mathrm{g}}^{2}=$ .01 (see Fig. 6). This significant interaction was because increasing set size had an underadditive effect on T2 RT with SOA; at the long SOA, increasing set size significantly lengthened T2 RT by $69 \mathrm{~ms}, p<.01$, whereas there was no significant set size effect at the short SOA (12 ms difference, $p>$ 17). The analysis of T1 RT revealed a significant main effect of SOA, $F(1,13)=68.16, p<.01, \eta_{\mathrm{g}}^{2}=.55$, with no other main effect and interaction.

Notably, this was a contrasting result with that of Experiment 1 . Specifically, when participants searched for a specific orientation target among heterogeneous distractors, set size had an additive effect on search performance with SOA. However, increasing the set size of a conjunction search had a different effect; search RT increased only at the long SOA, but not at the short SOA. To quantify this differential pattern across search tasks, I ran a three-way mixed ANOVA with set size (Set Size 3 vs. Set Size 6) and SOA (short vs.

Table 2 RT (ms) and accuracy (\%, in parentheses) data for Experiment 2

Task 2 (visual search task)

\begin{tabular}{lll} 
& Task 2 difficulty & \\
SOA & Set Size 3 & Set Size 6 \\
Short & $1,004(93.0)$ & $1,016(87.8)$ \\
Long & $745(96.7)$ & $814(92.3)$ \\
Task 1 (four-choice letter task) & \\
& Task 2 difficulty & Set Size 6 \\
SOA & Set Size 3 & $843(96.7)$ \\
Short & $831(97.5)$ & $586(97.1)$ \\
Long & $577(96.1)$ & \\
\hline
\end{tabular}

long) as within subject factors and search type (orientation search of Exp. 1 vs. conjunction search of Exp. 1) as a between-subjects factor. This analysis revealed a significant three-way interaction, $F(1,30)=5.78, p<.05, \eta_{\mathrm{g}}^{2}=.002$, confirming that increasing set sizes of the orientation and conjunction searches differentially interacted with the SOA manipulation.

As I predicted, when the search items were processed in parallel, increasing the search set size affected search RT only at the long SOA, but not at the short SOA. This is a replication of the results that Reimer and colleagues reported. These results indicate that in a dual-task situation, increasing the set

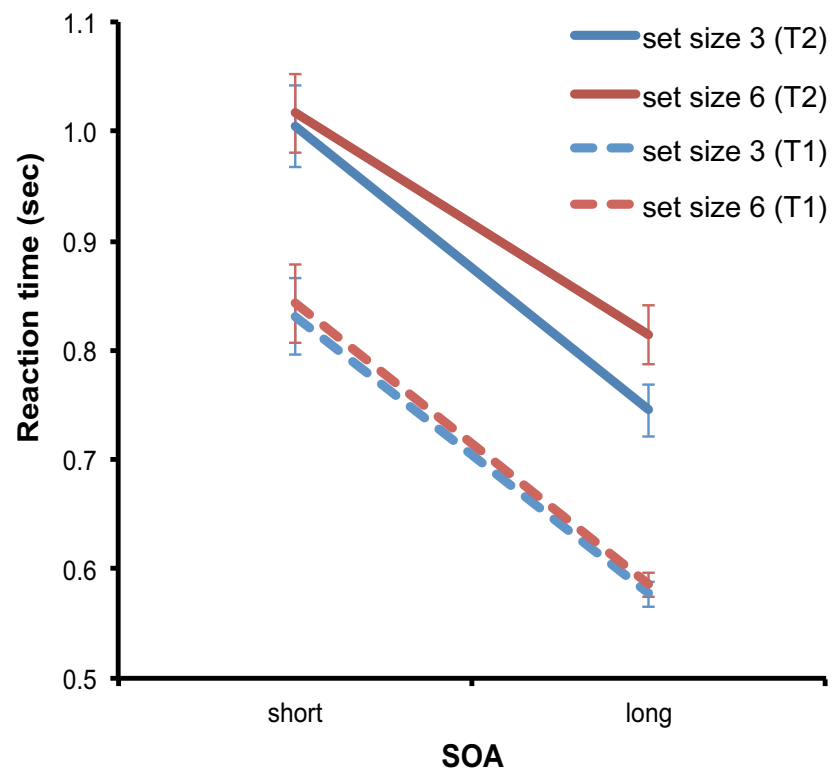

Fig. 6 Results of Experiment 2. Error bars represent standard errors of the mean. $\mathrm{T} 1=$ Task $1 ; \mathrm{T} 2=$ Task 2 (Color figure online) 
size of a visual search can have an additive or underadditive effect with SOA on search RT, depending on the nature of search process. If the search is parallel, an underadditive interaction between set size and SOA should be found. By contrast, with a serial search, the increased search set size should increase the demand for a process mediated by central attention, the serial deployment of focal attention, yielding an additive interaction between set size and SOA.

To be noted, the magnitude of set size effect at the long SOA was comparable to that of the orientation search task in Experiment 1. As elaborated in the introduction, this significant search slope by itself does not directly tell whether the search is serial or parallel; the limited parallel search model, positing that the finite amount of attentional resource is distributed among search items, predicts the exactly same magnitude of set size effect as the serial model does (Sung, 2008; Townsend, 1971, 1972).

\section{General discussion}

This study investigated how central attention interacts with perceptual attention. In two experiments reported here, a task taxing response selection process at the central stage (fouralternative choice task) was followed by a perceptually demanding search task with the short or long SOA. As a result, when the search stimuli were degraded by lowering their contrast, the search RT was lengthened only at the long SOA, but not at the short SOA. This underadditive interaction of SOA and perceptual difficulty on search RT suggests that the central process of a stimulus can be done in parallel with the perceptual process of another stimulus. By contrast, when the search process became demanding as search set size increased, an additive effect of search difficulty and SOA was found. However, such an additive effect was found only when the search process heavily required the serial shifting of focal attention.

The nature of relationship between central and perceptual attention has been controversial; while some studies showed that perceptual and central attention had separate capacities (Pashler, 1991; Pashler \& Johnston, 1989; Reimer et al., 2015), others showed evidence for a close tie between the two (Brisson \& Jolicoeur, 2007a, b; Robitaille et al., 2007). An important contribution of this study is that it clarified when perceptual and central attention are independent and when they interact with each other. Based on the present results and other previous findings, I suggest that central attention and perceptual attention are, by default, separate mechanisms. However, there are some circumstances when they interact. That is when the limited perceptual capacity is strained to the extent that only a subset of inputs can be processed at a time. In this case, central attention comes into play to resolve this limitation by serially allocating focal attention to the stimuli.
While increasing the set size of the orientation search task in Experiment 1 had an additive effect on search RT with SOA, the similar manipulation in the conjunction search task in Experiment 2 yielded an underadditive interaction between set size and SOA. This should be because each type of searches strained the perceptual capacity in different manners. Specifically, the process of searching for a specific orientation target among distractors of two different orientations in Experiment 1 is known to depend on serial search even though the target could be readily discriminable from the distractors (Wolfe et al., 1992; Woodman \& Luck, 1999). To locate a specific orientation target among multiple, heterogeneous distractors, focal attention should be serially allocated to each search stimulus.

By contrast, searching for a conjunction target can be done in a different manner. The analyses of simple features of multiple stimuli, such as color, shape, or orientation, can proceed in parallel, followed by the deployment of focal attention toward the feature conjunction target (Bichot et al., 2005). Specifically, as an anonymous reviewer, detecting a feature conjunction might comprise several distinct processes. For example, in the process of searching for a red tilted grating among red vertical and green tilted gratings, initially, the locations containing the red items should be tagged for further guidance of focal attention. This location tagging process might proceed in a parallel manner at the perceptual stage, consuming the limited amount of attentional resource. While this perceptual process can proceed simultaneously with a central process of the concurrent task, capacity-limited perceptual attention should be needed. This account can explain both the significant set size effect of the conjunction search at the long SOA and the underadditive effect of set size and SOA on search RT with SOA.

The framework proposed in the present study not only helps resolve the longstanding issue of the relationship between central and perceptual attention but also fits well with recent findings from cognitive neuroscience research. As mentioned earlier, Brisson and Jolicoeur showed that concurrent response selection load impaired the deployment of visuospatial attention required for the performance of a visual search task (Brisson \& Jolicoeur, 2007a, b). In line with this, a brain region associated with response selection, the lateral prefrontal cortex (LPFC) or inferior frontal junction (IFJ), was found to be activated when focal attention was allocated to a specific location (Asplund, Todd, Snyder, \& Marois, 2010). Furthermore, the IFJ was implicated in a unified, shared bottleneck responsible for the psychological refractory period and the attentional blink (Marti, Sigman, \& Dehaene, 2012; Tombu et al., 2011).

Finally, a neuroimaging study showed that the manipulation of increasing the number of stimuli to be processed increased activation of the IFJ, but the manipulation of degrading the quality of sensory inputs did not (Han \& 
Marois, 2013a). Notably, both types of task difficulty manipulations impaired behavioral performance similarly, but only the former increased the IFJ activation. This imaging data demonstrated a neural dissociation between capacity limits imposed on the information processing system and limits of quality of sensory data inputted to the system (Garner, 1970; Lavie \& de Fockert, 2003; Norman \& Bobrow, 1975). Considering this finding, together with the studies mentioned above, one can infer that the IFJ is involved in both response selection process and resolving capacity limitations by shifting attention. This clearly explains why concurrent response selection load delays visual search when search set size increased in the present study. Specifically, imagine that a search task taxing the perceptual capacity should be performed while response selection proceeds. To resolve the perceptual capacity limit, focal attention should be serially deployed, which is mediated by the IFJ. However, when this region is already engaged in the response selection process, the search process should be postponed until the response selection process finishes, yielding an additive effect of set size with SOA on search RT.

To conclude, this study showed that task demands affect the relationship between central and perception attention. By default, central attention is separate from perceptual processing, such that the perceptual analysis of a stimulus can proceed simultaneously with the central operation of another stimulus without mutual interference. However, when the number of items to be examined increases, thereby increasing the demand for serial shifting of focal attention, central attention comes into play to resolve this situation. What matters here is not just the set size, but whether increasing set size also increases the demand for serial shifting. When multiple items can be processed in parallel, the set size manipulation did not evoke the involvement of central attention in visual search.

\section{References}

Asplund, C. L., Todd, J. J., Snyder, A. P., \& Marois, R. (2010). A central role for the lateral prefrontal cortex in goal-directed and stimulusdriven attention. Nature Neuroscience, 13, 507-513.

Bichot, N. P., Rossi, A. F., \& Desimone, R. (2005). Parallel and serial neural mechanisms for visual search in macaque area V4. Science, 308(5721), 529-534. doi:10.1126/science.1109676

Bouvier, S., \& Treisman, A. (2010). Visual feature binding requires reentry. Psychological Science, 21(2), 200-204. doi:10.1177/0956797609357858

Brisson, B., \& Jolicoeur, P. (2007a). Electrophysiological evidence of central interference in the control of visuospatial attention. Psychonomic Bulletin \& Review, 14, 126-132.

Brisson, B., \& Jolicoeur, P. (2007b). A psychological refractory period in access to visual short-term memory and the deployment of visual-spatial attention: Multitasking processing deficits revealed by event-related potentials. Psychophysiology, 44(2), 323-333. doi:10.1111/j.1469-8986.2007.00503.x
Buschman, T. J., \& Miller, E. K. (2009). Serial, covert shifts of attention during visual search are reflected by the frontal eye fields and correlated with population oscillations. Neuron, 63(3), 386-396. doi:10.1016/j.neuron.2009.06.020

Carrasco, M., Penpeci-Talgar, C., \& Eckstein, M. (2000). Spatial covert attention increases contrast sensitivity across the CSF: Support for signal enhancement. Vision Research, 40(10/12), 1203-1215.

Cohen, M. A., Cavanagh, P., Chun, M. M., \& Nakayama, K. (2012). The attentional requirements of consciousness. Trends in Cognitive Science, 16(8), 411-417. doi:10.1016/j.tics.2012.06.013

Desimone, R., \& Duncan, J. (1995). Neural mechanisms of selective visual attention. Annual Review of Neuroscience, 18, 193-222.

Dosher, B. A., \& Lu, Z. L. (2000). Mechanisms of perceptual attention in precuing of location. Vision Research, 40, 1269-1292.

Garner, W. R. (1970). The stimulus in information processing. American Psychologist, 25, 350-358.

Han, S. W., \& Marois, R. (2013a). Dissociation between process-based and data-based limitations for conscious perception in the human brain. NeuroImage, 64, 399-406. doi:10.1016/j.neuroimage.2012.09.016

Han, S. W., \& Marois, R. (2013b). The source of dual-task limitations: Serial or parallel processing of multiple response selections? Attention, Percept ion, \& Psychophysics, 75(7), 1395-1405. doi:10.3758/s13414-013-0513-2

Han, S. W., \& Marois, R. (2014). The effects of stimulus-driven competition and task set on involuntary attention. Journal of Vision, 14(7). doi:10.1167/14.7.14

Huang, L., \& Pashler, H. (2005). Attention capacity and task difficulty in visual search. Cognition, 94(3), B101-B111. doi:10.1016/j. cognition.2004.06.006

Johnston, J. C., \& McCann, R. S. (2006). On the locus of dual-task interference: Is there a bottleneck at the stimulus classification stage? Quarterly Journal of Experimental Psychology, 59(4), 694-719. doi:10.1080/02724980543000015

Johnston, J. C., McCann, R. S., \& Remington, R. W. (1995). Chronometric evidence for two types of attention. Psychological Science, 6(6), 365-369. doi:10.1111/j.1467-9280.1995.tb00527.x

Jolicoeur, P., \& Dell'Acqua, R. (1998). The demonstration of shortterm consolidation. Cognitive Psychology, 36(2), 138-202. doi:10.1006/cogp.1998.0684

Lavie, N., \& de Fockert, J. W. (2003). Contrasting effects of sensory limits and capacity limits in visual selective attention. Perception \& Psychophysics, 65(2), 202-212.

Lien, M.-C., Croswaite, K., \& Ruthruff, E. (2011). Controlling spatial attention without central attentional resources: Evidence from event-related potentials. Visual Cognition, 19(1), 37-78.

Luck, S. J., \& Ford, M. A. (1998). On the role of selective attention in visual perception. Proceedings of National Academy of Sciences, 95, 825-830.

Marois, R., \& Ivanoff, J. (2005). Capacity limits of information processing in the brain. Trends in Cognitive Science, 9(6), 296-305. doi:10.1016/j.tics.2005.04.010

Marti, S., Sigman, M., \& Dehaene, S. (2012). A shared cortical bottleneck underlying attentional blink and psychological refractory period. NeuroImage, 59(3), 2883-2898. doi:10.1016/j.neuroimage.2011.09.063

McCann, R. S., Remington, R. W., \& Van Selst, M. (2000). A dual-task investigation of automaticity in visual word processing. Journal of Experimental Psychology:Human Perception \& Performance, 26(4), 1352-1370.

Miller, J., Ulrich, R., \& Rolke, B. (2009). On the optimality of serial and parallel processing in the psychological refractory period paradigm: Effects of the distribution of stimulus onset asynchronies. Cognitive Psychology, 58(3), 273-310. doi:10.1016/j.cogpsych.2006.08.003

Norman, D. A., \& Bobrow, D. G. (1975). On data-limited and resourcelimited processes. Cognitive Psychology, 4, 44-64. 
Pashler, H. (1984). Processing stages in overlapping tasks: Evidence for a central bottleneck. Journal of Experimental Psychology:Human Perception \& Performance, 10(3), 358-377.

Pashler, H. (1991). Shifting visual attention and selecting motor responses: Distinct attentional mechanisms. Journal of Experimental Psychology: Human Perception and Performance, 17, 1023-1040.

Pashler, H. (1994a). Dual-task interference in simple tasks: Data and theory. Psychological Bulletin, 116(2), 220-244.

Pashler, H. (1994b). Graded capacity-sharing in dual-task interference? Journal of Experimental Psychology: Human Perception and Performance, 20, 330-342.

Pashler, H., \& Johnston, J. C. (1989). Chronometric evidence for central postponement in temporally overlapping tasks. Quarterly Journal of Experimental Psychology Section A: Human Experimental Psychology, 41(1), 19-45.

Peirce, J. W. (2007). PsychoPy-Psychophysics software in Python. Journal of Neuroscience Methods, 162(1/2), 8-13. doi:10.1016/j. jneumeth.2006.11.017

Prinzmetal, W., McCool, C., \& Park, S. (2005). Attention: Reaction time and accuracy reveal different mechanisms. Journal of Experimental Psychology: General, 134, 73-92.

Reimer, C. B., Strobach, T., Frensch, P. A., \& Schubert, T. (2015). Are processing limitations of visual attention and response selection subject to the same bottleneck in dual-tasks? Attention, Perception, \& Psychophysics, 77(4), 1052-1069. doi:10.3758/s13414-0150874-9

Robitaille, N., Jolicoeur, P., Dell'Acqua, R., \& Sessa, P. (2007). Short-term consolidation of visual patterns interferes with visuo-spatial attention: Converging evidence from human electrophysiology. Brain Research, 1185, 158-169. doi:10.1016/j.brainres.2007.09.004

Ruthruff, E., Miller, J., \& Lachmann, T. (1995). Does mental rotation require central mechanisms? Journal of Experimental Psychology: Human Perception \& Performance, 21(3), 552-570.

Schweickert, R. (1980). Critical-path scheduling of mental processes in a dual task. Science, 209(4457), 704-706.

Sung, K. (2008). Serial and parallel attentive visual searches: Evidence from cumulative distribution functions of response times. Journal of Experimental Psychology: Human Perception \& Performance, 34(6), 1372-1388. doi:10.1037/a0011852
Tombu, M. N., Asplund, C. L., Dux, P. E., Godwin, D., Martin, J. W., \& Marois, R. (2011). A Unified attentional bottleneck in the human brain. Proceedings of the National Academy of Sciences of the United States of America, 108(33), 13426-13431. doi:10.1073/pnas.1103583108

Tombu, M., \& Jolicoeur, P. (2003). A central capacity sharing model of dual-task performance. Journal of Experimental Psychology: Human Perception \& Performance, 29(1), 3-18.

Tombu, M., \& Jolicoeur, P. (2005). Testing the predictions of the central capacity sharing model. Journal of Experimental Psychology:Human Perception \& Performance, 31(4), 790802. doi:10.1037/0096-1523.31.4.790

Townsend, J. T. (1971). A note on the identifiability of parallel and serial processes. Perception \& Psychophysics, 10(3), 161-163.

Townsend, J. T. (1972). Some results concerning the identifiability of parallel and serial processes. British Journal of Mathematical and Statistical Psychology, 25(2), 168-199.

Treisman, A. M., \& Gelade, G. (1980). A feature-integration theory of attention. Cognitive Psychology, 12(1), 97-136.

Ulrich, R., \& Miller, J. (2008). Response grouping in the psychological refractory period (PRP) paradigm: Models and contamination effects. Cognitive Psychology, 57(2), 75-121. doi:10.1016/j. cogpsych.2007.06.004

Welford, A. T. (1952). The 'psychological refractory period' and the timing of high-speed performance- a review and a theory. British Journal of Psychology, 43, 2-19.

Wolfe, J. M., Cave, K. R., \& Franzel, S. L. (1989). Guided search: An alternative to the feature integration model for visual search. Journal of Experimental Psychology:Human Perception \& Performance, 15(3), 419-433.

Wolfe, J. M., Friedman-Hill, S. R., Stewart, M. I., \& O'Connell, K. M. (1992). The role of categorization in visual search for orientation. Journal of Experimental Psychology:Human Perception \& Performance, 18(1), 34-49.

Woodman, G. F., \& Luck, S. J. (1999). Electrophysiological measurement of rapid shifts of attention during visual search. Nature, 400(6747), 867-869. doi:10.1038/23698 